\title{
Carnets
}

Revue électronique d'études françaises de l'APEF

Deuxième série - 8 | 2016

Du Français en cause aux causes du français

\section{La langue française À l'université algérienne : changement de statut et impact}

\section{Lamia Boukhannouche}

\section{OpenEdition}

1 Journals

\section{Édition électronique}

URL : https://journals.openedition.org/carnets/1895

DOI : 10.4000/carnets. 1895

ISSN : 1646-7698

Éditeur

APEF

\section{Référence électronique}

Lamia Boukhannouche, "La langue française À l'université algérienne : changement de statut et impact », Carnets [En ligne], Deuxième série - 8 | 2016, mis en ligne le 30 novembre 2016, consulté le 21 septembre 2021. URL : http://journals.openedition.org/carnets/1895 ; DOI : https://doi.org/ $10.4000 /$ carnets. 1895

Ce document a été généré automatiquement le 21 septembre 2021.

\section{(ब) $(1) \otimes$}

Carnets est mis à disposition selon les termes de la licence Creative Commons - Atribution - Pas d'utilisation commerciale 4.0 International. 


\title{
La langue française À l'université algérienne : changement de statut et impact
}

\author{
Lamia Boukhannouche
}

\section{Introduction}

1 Le français reste présent en Algérie en occupant le rôle de la langue d'information, de communication et de fonctionnement de diverses institutions de l'État. Autrement-dit, les médias, certains secteurs économiques, le système éducatif et notamment l'université sont les lieux privilégiés de la langue française. À l'université algérienne, le français tient une position forte dans l'enseignement scientifique et technique (sciences médicales, sciences de l'ingénieur, etc.). À ce propos, le constat que fait Achouche reste d'actualité : "Malgré l'indépendance et les actions d'arabisation qui s'en sont suivies, les positions du français n'ont pas été ébranlées, loin de là, son étude ayant même quantitativement progressé du fait de sa place dans l'actuel système éducatif algérien» (Achouche, 1981: 46).

2 L'enseignement du primaire jusqu'au secondaire est dispensé en arabe littéraire moderne et ce n'est qu'à partir de la deuxième année de l'enseignement primaire que l'apprentissage du français apparait dans les programmes. Cela signifie que l'arabe a pris en charge les enseignements de l'ensemble des matières du primaire jusqu'au secondaire. À l'université, cette arabisation (1970) n'a été poursuivie que dans certaines disciplines universitaires, sciences sociales, économiques, commerciales... Et la plupart des filières scientifiques et techniques telles que médecine, sciences vétérinaires, pharmacie, architecture... enseignent en français. Le français devient donc langue d'accès à la formation scientifique.

3 Nous sommes face à deux contextes d'enseignement/apprentissage du français différents, le secondaire d'un côté et le supérieur d'un autre. Queffélec et Derradji (2002) présentent 
l'enseignement du français en Algérie comme un enseignement du type institutionnel formel, assuré par des structures éducatives scolaires et universitaires ${ }^{1}$.

\section{La place du français dans l'enseignement algérien : entre le secondaire et le supérieur algérien (à partir de 1970)}

\section{Le secondaire}

On peut déterminer à la fois le statut de la langue ainsi que la valeur qui lui est consacrée, en observant les objectifs qui lui sont assignés ainsi que les choix méthodologiques qui sous-tendent son apprentissage. Ainsi, l'éducation nationale stipule que l'enseignement/ apprentissage du français en Algérie est censé se fonder sur l'acquisition d'un moyen de communication qui permet l'accès au savoir, à partir de l'utilisation des nouvelles technologies, de la familiarisation avec la sphère culturelle francophone, et aussi de l'ouverture d'esprit sur le monde.

5 Nous constatons que nous avons des objectifs qui relèvent de deux ordres distincts, à savoir de l'ordre humaniste qui se caractérise par l'ouverture sur d'autres cultures étrangères ainsi que sur les nouvelles technologies du monde et de l'ordre intellectuel qui porte essentiellement sur l'utilisation efficace de l'outil linguistique permettant l'accès au savoir. Cela dit, nous nous sentons plus concernés par le dernier objectif puisqu'il rentre directement dans le cadre de notre étude. Un objectif qui permet aux apprenants, donc futurs étudiants à la fin de leur parcours de bénéficier d'une maîtrise linguistique suffisante leur permettant de réussir leur formation universitaire. A ce titre, des manuels ont été conçus et l'objet de notre étude en est un exemple représentatif :

- Manuels 1 (1982-1983): ces derniers traitent des thématiques assez variées telles que : famille, école, problèmes sociaux, etc. Le matériel utilisé porte sur des textes dont la plupart sont extraits d'œuvres littéraires d'auteurs d'expression française qui donnent la priorité à l'aspect socio-culturel. Quant aux activités, nous avons celles de compréhension écrite, d'expression écrite et de renforcement linguistique.

- Manuels 2 (1988-1989) : dans ce genre de manuels, on remarque que les situations relevant essentiellement du domaine scientifique et technique y sont souvent présentes. En effet, les concepteurs ont opté pour des textes extraits d'œuvres de revues scientifiques réalisées par des auteurs algériens. Quant aux activités, on assiste à la présence d'exercices de compréhension et d'expression (écrite) et d'exercices structuraux (lexique, morphologie, etc.)

6 La description ci-dessus montre clairement que les manuels 1 préconisent un enseignement basé essentiellement sur des écrits littéraires quant aux manuels 2, ceux-ci s'appuient en revanche sur le domaine scientifique et technique. Nous remarquons donc qu'il y a un réel changement d'orientation en matière d'enseignement de la langue dont les principes relèvent finalement d'une nouvelle méthodologie et d'un autre type d'éducation. Les thèmes ont été choisis de façon à diriger les apprenants vers un modèle d'apprentissage bien déterminé, à savoir un modèle pour l'acquisition de réelles compétences scientifiques et techniques. Cependant, malgré les grands efforts réalisés par les officiels de changer les orientations pédagogiques à travers les manuels scolaires 
pour le français au secondaire, cela les a confrontés à une situation potentiellement délicate incitant encore une fois à de nouvelles réformes.

- Manuels 3 (1990-2003) : durant ces années-là, les premiers réaménagements des programmes ont commencé à voir le jour. Cependant, on assiste tout de même à l'absence totale de toute créativité, recherche ou encore innovation dans le milieu scolaire. Les manuels du secondaire portent sur des textes soit «fabriqués " par les auteurs des manuels, soit tirés de sources diverses (article, poème...). Les manuels se donnent par exemple pour mission d'apprendre essentiellement à lire mais pas d'une manière autonome.

7 Ainsi, l'enseignement du français connaît un renouvellement grâce aux innovations apportées par de nouvelles implications didactiques et pédagogiques.

- Manuels 4 (de 2003 jusqu'à aujourd'hui) : ce type de manuels préconise l'utilisation des textes authentiques (extraits d'œuvres littéraires, articles de presse, publicités...). L'objectif est la découverte de la cohérence interne d'un texte en mettant en place de réelles compétences autonomes de lecture. Quant aux activités, on remarque que la grammaire, la conjugaison, le vocabulaire et l'orthographe sont désormais liés à des objectifs définis. En ce qui concerne la dimension scripturale, avec les nouveaux programmes on se préoccupe assez du processus de construction: en favorisant par exemple l'écriture de reformulation ou l'écriture d'invention.

8 Au-delà de toutes ces réformes qui ont tenté d'apporter un certain renouveau à l'enseignement du français dans le secondaire à travers les différents manuels scolaires, la langue (le français) continue à subir dans le système éducatif algérien, en l'occurrence, dans le contexte universitaire, de réels changements.

\section{L'université}

9 Avec la politique de l'arabisation (1970), la langue française change complètement de statut. On passe du français comme première langue étrangère étudiée et enseignée pour elle-même à un français qui devient un moyen de réussite, c'est-à-dire, d'un statut de discipline enseignée jusqu'au secondaire, à celui de langue d'enseignement des sciences et des techniques dans le supérieur². Cet état de fait s'avère assez problématique pour les nouveaux arrivants à l'université qui se retrouvent contraints de poursuivre des études complexes dans une langue que très souvent ils ne maîtrisent pas suffisamment.

En effet, la rupture avec la formation antérieure des élèves avant leur arrivée à l'université est bien réelle et les objectifs accordés à l'enseignement du français dans le secondaire ne répondent pas forcément aux besoins de l'université à cause du changement de langue d'enseignement. Cette situation a certes conduit à une certaine prise en charge d'un point de vue didactique grâce aux dispositifs de cours de français mis en place par l'institution dans la majorité des disciplines scientifiques et techniques au supérieur telles que : les sciences vétérinaires (avec 30h de cours par semestre) mais cela reste remarquablement insuffisant.

11 À l'université, nous sommes donc face à une situation où on voit les nouveaux inscrits essentiellement ceux des disciplines scientifiques et techniques en français, en début de leur formation universitaire être confrontés à un double enseignement: il s'agit d'un enseignement de français (présenté sous forme de cours de langue) au-delà de l'enseignement en français (qui concerne les cours de la discipline). 


\section{Cours EN français vs Cours DE français à l'université algérienne (réforme de 2004)}

Malgré la réforme qui a touché le supérieur algérien en 2004, et qui portait sur ce que l'on appelle aujourd'hui le système LMD (Licence, Master, Doctorat) ${ }^{3}$, cette situation n'a pas empêché de procurer à la langue française un double statut qui conduit logiquement à deux types de cours: cours de français mis en œuvre pour l'amélioration des compétences linguistiques des étudiants et cours en français qui concerne leur réussite dans la discipline choisie.

\section{Cours EN français}

13 L'université algérienne offre une panoplie de formations en français assez importante aux étudiants ayant accès au supérieur, comme les sciences médicales (pharmacie, médecine, etc.) ou encore les filières techniques (informatique, architecture, etc.). Le français a donc le statut de véhicule d'enseignement à travers les cours EN français. Nous tentons de représenter au mieux les contenus et le volume horaire existant dans l'échantillon des filières scientifiques et techniques enseignées en langue française à l'université de Blida que nous avons choisi pour notre étude. Blida est prise seulement comme exemple afin de mieux illustrer le phénomène :

\begin{tabular}{|l|l|l|l|}
\hline Filières & $\begin{array}{l}\text { Nombre des modules } \\
\text { enseignés en français }\end{array}$ & $\begin{array}{l}\text { Brève présentation de la } \\
\text { filière }\end{array}$ & $\begin{array}{l}\text { Volume horaire } \\
\text { consacré }\end{array}$ \\
\hline $\begin{array}{l}\text { Sciences } \\
\text { vétérinaires }\end{array}$ & 35 modules & Ingénierie & $\begin{array}{l}\text { Environ } \\
\text { semestrielles }\end{array}$ \\
\hline Biologie & $\begin{array}{l}\text { modules pour } \\
\text { chacune des spécialités }\end{array}$ & $\begin{array}{l}\text { Licence de 3ans avec } 8 \\
\text { spécialités } \\
\text { différentes }\end{array}$ & $\begin{array}{l}\text { Environ } \\
\text { semestrielles }\end{array}$ \\
\hline $\begin{array}{l}\text { Chirurgie } \\
\text { dentaire }\end{array}$ & 45 modules & $\begin{array}{l}\text { Ingénierie (la 1ère année est } \\
\text { un tronc commun médical) }\end{array}$ & $300 h$ semestrielles \\
\hline Génie civil & $\begin{array}{l}30 \text { modules pour chaque } \\
\text { spécialité }\end{array}$ & $\begin{array}{l}\text { Ingénierie : la filière propose } \\
\text { deux } \\
\text { options : géotechnique/ } \\
\text { structures et matériaux }\end{array}$ & 350h semestrielles \\
\hline
\end{tabular}

Ces filières représentent le choix le plus prisé des nouveaux inscrits. Généralement, une bonne moyenne à l'examen du baccalauréat est requise pour l'admission dans ces filières. Ces dernières, nous le constatons dans le tableau ci-dessus consacrent un nombre de modules et un volume horaire assez importants. Il arrive aussi que les filières en question connaissent un taux d'échec énorme surtout en $1^{\text {ère }}$ année. L'étudiant dès son arrivée universitaire connaît des problèmes qui relèvent de la maîtrise langagière mais aussi des difficultés à s'adapter à son nouvel environnement, à se prendre en charge 
ultérieurement, à acquérir ainsi une autonomie de gestion de sa vie quotidienne et surtout une autonomie pédagogique et de gestion de sa formation.

\section{Cours DE français}

Dans la perspective de régler les problèmes auxquels les étudiants se heurtent dès leur arrivée à l'université, les instructions officielles ont jugé essentiel de mettre en place un dispositif de renforcement linguistique en langue française en début de cursus de certaines filières scientifiques et techniques francophones. On assiste donc à l'enseignement du français comme matière, incluse dans le programme de la formation au même titre que les autres matières de spécialité. Autrement-dit, le français dans ce contexte, continue à être langue objet d'enseignement comme dans les autres paliers de l'éducation nationale même si le volume horaire et les contenus enseignés sont différents.

\section{Dispositifs existants à l'université algérienne pour les filières scientifiques et techniques (de 2004 jusqu'à aujourd'hui)}

Dans certaines disciplines scientifiques et techniques, le français n'est enseigné qu'en première année universitaire ${ }^{4}$ pour un volume horaire ne dépassant pas les $3 \mathrm{~h}$ hebdomadaires. Dans le système Licence-Mastère-Doctorat (LMD), la majorité des filières scientifiques et techniques consacrent un volume horaire de $30 \mathrm{~h}$ au module de français. Tel est le cas des sciences vétérinaires, biologie, chirurgie dentaire, agronomie, génie civil, etc. Les contenus enseignent une langue spécialisée qui se caractérise généralement par l'usage du minimum terminologique et phraséologique nécessaire à la production et à la réception d'un discours spécialisé cohérent et juste en français. Ci-dessous, nous avons un tableau décrivant les cours de français dans certaines filières scientifiques et techniques enseignées à l'université de Blida :

\begin{tabular}{|l|l|l|l|l|}
\hline $\begin{array}{l}\text { Cours de } \\
\text { français } \\
\text { Filières }\end{array}$ & $\begin{array}{l}\text { Intitulé du } \\
\text { cours }\end{array}$ & $\begin{array}{l}\text { Volume } \\
\text { horaire }\end{array}$ & Contenu du cours & $\begin{array}{l}\text { Evaluation } \\
\text { du cours }\end{array}$ \\
\hline $\begin{array}{l}\text { Sciences } \\
\text { vétérinaires }\end{array}$ & Terminologie & $\begin{array}{l}30 \mathrm{~h} \text { pour le } \\
\text { semestre }\end{array}$ & $\begin{array}{l}\text { Activités sur : orthographe, } \\
\text { conjugaison, lexique en lien } \\
\text { avec la discipline }\end{array}$ & semestrielle \\
\hline Biologie & Français & $\begin{array}{l}30 h \text { pour le } \\
\text { semestre 1 }\end{array}$ & Prise de notes, étude de textes & Idem \\
\hline $\begin{array}{l}\text { Chirurgie } \\
\text { dentaire }\end{array}$ & Langue I & $\begin{array}{l}30 h \text { pour le } \\
\text { semestre 1 }\end{array}$ & Lecture/compréhension & Idem \\
\hline Génie civil & Langue & $\begin{array}{l}22,5 h \text { pour le } \\
\text { semestre 1 }\end{array}$ & Lecture des textes & Idem \\
\hline
\end{tabular}


$17 \mathrm{Si}$ on regarde le tableau 2 en comparaison avec le tableau 1 et sous des termes différents, on remarque que les disciplines citées en haut s'accordent à travailler dans le module de «langue ", qu'ils appellent dans la majorité des cas «français ", la lecture et l'étude de textes aussi bien pendant les séances de TD qu'en évaluation finale. On se rend compte aussi que le volume horaire accordé au module de français est considérablement pauvre par rapport aux besoins des étudiants. Pour mieux illustrer la situation, nous présentons en guise d'exemple les contenus d'un cours DE français d'une discipline scientifique afin de définir la relation établie entre ces derniers et les cours disciplinaires. Nous avons choisi donc de passer en revue ce qui se fait en «sciences vétérinaires » en termes d'enseignement de la langue française.

\section{Cours DE français en sciences vétérinaires}

\section{Objets et enjeux}

18 Nous abordons ici la mise en œuvre du programme de renforcement linguistique en français, au département des sciences vétérinaires de l'université de Blida. Il s'agit d'un enseignement de langue étrangère destiné à des étudiants en cycle de graduation, pour l'obtention du diplôme d'Ingénieur d'Etat en sciences vétérinaires. Notre analyse du programme constitue une étape indispensable pour la réalisation de notre programme de formation adapté aux besoins de notre public en sciences vétérinaires.

\section{Description du contexte}

19 Le département des sciences vétérinaires de l'université de Blida forme des Docteurs en Médecine vétérinaire (DMV) et des spécialistes exerçant dans des filières aussi diverses que la production animale, l'hygiène et l'inspection des denrées animales et d'origine animale ainsi que dans l'industrie pharmaceutique ou la recherche appliquée. Le cursus pour l'obtention du diplôme de vétérinaire requiert cinq années d'études. Quant au module de français, il figure lors de la première année de la formation, à raison de trois heures hebdomadaires. Et il est désigné sous l'appellation « Terminologie ».

\section{Programme de français}

Lors de notre étape du recueil des données, nous avons pu nous procurer le programme et les contenus du cours de français (Manuel de terminologie, 2005) auprès de l'enseignante chargée de la matière, au département des sciences vétérinaires. Le renforcement linguistique en français qui est l'objet d'enseignement durant la première année du cursus est présenté sous forme de travaux dirigés (TD), avec un volume horaire hebdomadaire de 3 heures et un volume horaire semestriel de 30 heures. En vétérinaire, le module de français porte l'intitulé générique de "Terminologie » et l'institution lui consacre 10 TD pour chaque semestre et chacun de ce dernier porte sur un cours traitant généralement un point de langue (conjugaison, vocabulaire...). Voici une synthèse des cours de français dispensés en sciences vétérinaires :

\begin{tabular}{|l|l|l|l|}
\hline Cours & Contenus & Exercices & Supports \\
\hline
\end{tabular}




\begin{tabular}{|c|c|c|c|}
\hline Terminologie & Présentation du concept & $\begin{array}{l}\text { Questions à } \\
\text { réponses courtes et } \\
\text { ouvertes (CROQ) }\end{array}$ & Photos d'animaux \\
\hline $\begin{array}{ll}\text { Les } & \text { temps } \\
\text { simples } & \end{array}$ & $\begin{array}{l}\text { Définition du présent de } \\
\text { l'indicatif, de l'imparfait et } \\
\text { du futur simple }\end{array}$ & $\begin{array}{l}\text { Absence des } \\
\text { exercices }\end{array}$ & \\
\hline $\begin{array}{l}\text { Les préfixes et } \\
\text { suffixes }\end{array}$ & $\begin{array}{l}\text { Tableau regroupant les mots } \\
\text { grecs servant de préfixes et } \\
\text { de suffixes }\end{array}$ & & \\
\hline Terminologie & $\begin{array}{l}\text { Liste de mots présentés en } \\
\text { plusieurs catégories (male, } \\
\text { femelle, petit, famille, cri) } \\
\text { Définition des classes } \\
\text { animales (mammifères, } \\
\text { reptiles, poissons, insectes, } \\
\text { oiseaux, amphibiens) }\end{array}$ & Exercices à trous & $\begin{array}{l}\text { Présentation } \\
\text { iconique et légendée }\end{array}$ \\
\hline Orthographe & $\begin{array}{l}\text { La nature et le rôle des } \\
\text { préfixes }\end{array}$ & & \\
\hline Terminologie & $\begin{array}{l}\text { Définition de l'anatomie, du } \\
\text { squelette }\end{array}$ & $\begin{array}{l}\text { Absence des } \\
\text { exercices }\end{array}$ & $\begin{array}{l}\text { Photo du squelette d'un } \\
\text { être-humain et de sa } \\
\text { mâchoire supérieure } \\
\text { légendée } \\
\text { Photo légendée du } \\
\text { squelette d'un cheval }\end{array}$ \\
\hline $\begin{array}{l}\text { Substituts } \\
\text { grammaticaux }\end{array}$ & $\begin{array}{l}\text { Définition et présentation } \\
\text { sous forme } \\
\text { de tableau: liste des } \\
\text { pronoms personnels, } \\
\text { possessifs et démonstratifs }\end{array}$ & $\begin{array}{l}\text { Exercices sur les } \\
\text { adjectifs possessifs, } \\
\text { auxiliaires et } \\
\text { conjonctions de } \\
\text { coordination (a/à, } \\
\text { est/et) }\end{array}$ & \\
\hline
\end{tabular}

21 Suite au tableau présenté ci-dessus, un échantillon de cours a été choisi en guise d'exemple que nous analyserons par la suite :

\section{Exemple 1}

\section{TERMINOLOGIE}

L'élevage : techniques par lesquelles on élève (des animaux domestiques ou utiles) en les faisant naître et se développer dans de bonnes conditions, en contrôlant leur entretien et leur reproduction, de manière à obtenir un résultat économique 
L'élevage des oiseaux, des volailles est.

L'élevage des lapins est.....

L'élevage des abeilles est

L'élevage des poissons est.

L'élevage des escargots est.

1) Donne le lieu d'habitation ou d'élevage des animaux suivants

Le chien : ................. Le canard :.................... Les abeilles :............ Le lapin :.................. Le pigeon : ... Le porc : Le cheval Les poules L'autruche :

Les oiseaux :................ Les poissons : Les fourmis :

Les moutons .. Les vaches

2) - Le récipient utilisé pour donner à manger aux animaux domestiques est. naturel ou aménagé ou les animaux vont boire est.

- L'espace aménage pour que les poules viennent y pondre est

- La cage ou le panier utilisé pour faire couver les oiseaux ou des volailles est

- Mâcher de nouveau des aliments revenus de l'estomac avant de les avaler définitivement.

\section{Exemple 2}

\section{FRANÇAIS \\ LES TEMPS SIMPLES}

\section{Le présent de l'indicatif}

Le présent exprime une action qui se fait au moment où l'on parle.

1) Verbes du premier groupe

$$
\text { e, es, e, ons, ez, ent }
$$

2) Verbes du second groupe

Les verbes du deuxième groupe suivent la règle générale

$$
s, s, t \text {, issons, issez, issent }
$$

3) Verbes du troisième groupe

$\mathrm{Au}$ présent de l'indicatif la plupart des verbes du troisième groupe ont les terminaisons suivantes : $\mathbf{s}, \mathbf{s}, \mathbf{t}$, ons, ez, ent

- Les verbes en dre comme perdre, coudre se terminent par :

$d s, d s, d$, ons, ez, ent

- sauf les verbes en ainder, eindre, oindre, soudre comme craindre, peindre, joindre résoudre qui eux suivent la règles générale $: s, s$, ons, ez, ent

- Les verbes pouvoir, vouloir valoir se terminent à l'écrit par : $x, x, t, o n s, e z$, ent

- Les verbes ouvrir, cueillir se terminent à l'écrit par $: e, e s, e$, ons, ez, ent

- Encore une petite exception avec vaincre qui garde son « $c$ »dans sa conjugaison et qui parfois se transforme en « qu »: cs, cs, c, quons, quez, quent

Les auxiliaires

Etre : je suis, tu es, il/elle est, nous sommes, vous êtes ils/ elles sont Avoir : j'ai, tu as, il/elle a, nous avons, vous avez, ils /elles ont 


\section{L'imparfait}

L’imparfait est un temps qui exprime le passé .C'est un temps qui exprime une durée indéfinie et qui nous sert à expliquer le décor de l'action, la répétition dans le passé, etc.

Pour la plupart des verbes, le radical de l'imparfait est la première personne du pluriel (nous du présent de l'indicatif

Les terminaisons sont : ais , ais, ait, ions, iez, aient

\section{Exemple 3}

\begin{tabular}{|l|}
\hline ORTHOGRAPHE \\
\hline Exercice 1 \\
\hline $\begin{array}{l}\text { a) Ajoute le préfixe « } \mathrm{r} \text { », « re », « ré » aux verbes pour obtenir la répétition de l'action : } \\
\text { - apparaître, ajouter, produire, ouvrir, absorber, aménager, inventer, partir, passer, venir, } \\
\text { transmettre, courir, donner, naître, abonner, }\end{array}$ \\
\hline $\begin{array}{l}\text { b) A l'aide des préfixes : contre, para, anti forme des mots exprimant l'opposition : } \\
\text { - tonnerre, tabac, sens, rouille, indication, thèse, chut, pluie, cœur, brouillard, cops, gène, } \\
\text { coup, mesure, champ, allergique }\end{array}$ \\
\hline $\begin{array}{l}\text { c)Ajoute l'un des préfixes : in, im ,dès, il , mé , ir, dé , pour les mots suivants afin d' obtenir son } \\
\text { contraire : } \\
\text { - correct, légal, régulier, agréable, connu, responsable, charger, espoir, habiller, localiser, } \\
\text { centraliser, lisible, poli, pardonnable, content. }\end{array}$
\end{tabular}

Le programme de renforcement linguistique en sciences vétérinaires présenté ci-dessus met en évidence quelques caractéristiques.

Le choix des contenus du programme de langue est étroitement lié au domaine des sciences vétérinaires. Nous retrouvons par exemple un cours de terminologie dont le thème est l'élevage. De nombreuses séances de vocabulaire sont proposées qui incitent à travailler davantage le lexique propre au domaine et dont l'étudiant vétérinaire a forcément besoin dans sa formation en cours. Nous retrouvons des verbes à conjuguer et des mots à construire à l'aide d'une affixation adéquate mais qui ne se rapportent pas exactement au domaine vétérinaire. Nous remarquons que les finalités assignées dans ce dispositif favorisent largement la maîtrise lexicale, syntaxique et orthographique de la langue au détriment de l'apprentissage des discours, en effet, nous constatons qu'il n'y a absolument rien en terme de discours (lus et écrits), les cours portent essentiellement sur les outils linguistiques. Nous constatons également la non détermination préalable des objectifs pédagogiques.

Quant aux activités proposées, elles sont présentées sous forme d'exercices structuraux. Et nous remarquons une vision réductrice de l'évaluation qui porte uniquement sur des lacunaires. Ce sont là autant d'indicateurs pertinents qui permettent d'offrir une vue d'ensemble propre à ce programme, en remarquant si en effet, existe-il une synergie entre l'enseignement spécifique souhaité, les contenus à enseigner et les activités pédagogiques proprement dites. 
Outre le vocabulaire de base de la spécialité, il est attendu du programme de français à l'intention des étudiants vétérinaires qu'il réponde aux besoins de ces derniers et à leurs préoccupations scientifiques telles que comprendre que ce soit à l'oral ou à l'écrit divers types d'énoncés de cours, maitriser l'expression écrite ou orale. Le programme est appelé à développer durant le cursus toutes ces compétences à même d'être réinvesties dans d'autres modules et dans leurs cursus vétérinaires. Il s'agit de plusieurs savoir-faire langagiers correspondant aux réels besoins du public et qui peuvent être aussi bien oraux que scripturaux.

\section{Proposition de programme de formation sur mesure pour les sciences vétérinaires}

Etant dans une perspective d'innovation pédagogique, nous nous sommes donné pour mission de faire une proposition de matériel pédagogique pour les sciences vétérinaires qui s'inscrit dans une démarche que l'on appelle aujourd'hui FOS/FOU (français sur objectif spécifique/français sur objectif universitaire). Il s'agit d'une approche qui est définie comme une formation de courte durée visant à développer chez le publicapprenant des compétences de communication professionnelle. Le FOS/FOU se donne pour but de se centrer sur des contenus a priori non maitrisés par l'enseignant d'où la nécessité d'entrer en contact avec les acteurs professionnels, d'élaborer le matériel et de didactiser des activités pédagogiques. Notre proposition de matériel s'est faite à partir d'un certain nombre de principes que nous allons citer comme suit :

\section{Enseignement de l'écrit : entre le quoi et le comment}

La finalité de notre étude est de mettre en œuvre une proposition didactique autour de laquelle nous avons construit des séquences pédagogiques (fiches-étudiant/fichesenseignant) comprenant deux éléments essentiels : le QUOI enseigner et le COMMENT enseigner.

On met derrière le "QUOI enseigner " tous les contenus pédagogiques à identifier et les objectifs à viser sans oublier les compétences langagières à faire acquérir par les étudiants. Il amène également à répondre aux questions suivantes : quels sont les savoirs qu'un étudiant doit acquérir? Que doit-il accomplir comme tâches? Quelles attitudes doit-il développer? Quant au « COMMENT enseigner », il permet de cibler une démarche à suivre pour un enseignement/apprentissage efficace. Il met en avant la façon de construire les connaissances et le chemin à suivre pour y parvenir.

\section{Savoir lire pour bien écrire}

Afin que les étudiants arrivent à s'approprier un écrit de leur spécialité, il faudrait que ces derniers s'appuient en premier lieu sur le savoir-lire. On finit par maitriser les règles qui sous-tendent un texte après avoir travaillé celui-ci en phase de lecture. On distingue donc une sorte de corrélation entre lecture et rédaction qui définit clairement qu'il y a moyen de reproduire un texte après l'avoir lu et étudié. La relation précise aussi la nécessité de se débarrasser de tous les problèmes qui relèvent de l'ordre de la compréhension avant de s'occuper de la forme du texte. 


\title{
Traitement des données collectées
}

\begin{abstract}
cours de notre étude. Ces données recueillies avaient besoin d'être retravaillées de façon à pouvoir les utiliser dans le programme. Il arrive parfois de tomber sur des données inexploitables d'où l'intérêt d'intervenir afin de les modifier, simplifier voire complexifier puisqu'il est recommandé de ne pas travailler sur l'objet final du document mais plutôt de mettre en place une progression d'enseignement.
\end{abstract}

\section{Le linguistique sans négliger le discursif}

31 Il est certes important de centrer toute son intention sur le «linguistique » dans la mesure où le problème de départ est a priori un problème de langue. Cependant, nous comptons inscrire notre conception du programme dans une perspective qui est fondée sur une approche reliant à la fois les aspects intra de la langue, autrement dit, (aspect syntaxique, lexical, etc.) aux aspects supra de la langue, à savoir les aspects contextuels (pragmatiques). Cette approche d'écriture prend en considération donc la jonction entre les unités fonctionnelles de la langue et le contexte de leur utilisation.

\section{Autonomie de l'apprentissage}

La conception du programme favorisera un apprentissage actif. La maitrise du savoir ne se fait pas par simple réception de la part de l'apprenant mais plutôt par tout un travail de construction et d'investissement personnel. Ce dernier possède des connaissances qu'il doit par la suite transformer. La méthode active n'a jamais considéré l'apprenant comme une page vierge que l'enseignement doit remplir mais une personne en complète évolution. L'apprenant construit ses connaissances à partir de ce qu'il sait auparavant et se met entre les vieilles et les nouvelles connaissances.

\section{Activités d'écriture}

Le programme linguistique préconise une approche par « modélisation » qui stipule, nous l'avons déjà mentionné, le passage de la compréhension à la production et c'est ce passage même qui doit être maitrisé à partir d'activités d'observation ou de repérage de textes. Ce sont des activités qui permettent l'identification du modèle d'écrit que les étudiants seront amenés à reproduire avec leur propre bagage langagier. L'entrainement à la modélisation continuera avec d'autres types d'activités rédactionnelles qui favorisent aussi bien l'écriture que la réécriture.

\section{En guise de conclusion}

Malgré cette place particulière qu'occupe la langue française dans le système éducatif algérien et la volonté de doter l'apprenant d'une compétence linguistique lui permettant de comprendre, de communiquer aisément et de poursuivre sa formation supérieure, sans oublier l'effort fourni par les officiels à vouloir pallier les problèmes liés à la langue en mettant en place des programmes de formation linguistique, la situation s'avère de 
plus en plus compliquée et suscite encore de nombreuses réflexions. Cette réalité interroge la didactique du français puisque la priorité est d'améliorer les compétences langagières et professionnelles des étudiants en l'occurrence, dans un contexte universitaire.

\section{BIBLIOGRAPHIE}

ACHOUCHE, Mohamed (1981). «La situation sociolinguistique en Algérie », Langues et Migrations, Grenoble : Presses Université de Grenoble.

BOUBAKouR, S (2008). » Étudier en français...Quelle histoire ! Le français en Afrique, Revue du Réseau des Observatoires du Français Contemporain en Afrique. Nice : Institut de linguistique française. $n^{\circ} 23$.

CORTIER, Claude et KAABOUB, Abdelkrim (2010). » Le français dans l'enseignement universitaire algérien : enjeux linguistiques et didactiques ». FDLM. Faire des études supérieures en langue française , Clé Internationale, no 47, pp. 53-63.

MANGIANTE, Jean-Marc, PARPETTE, Chantal (2004). Le Français sur objectifs spécifiques: de l'analyse des besoins à l'élaboration d'un cours. France : Hachette.

MANGIANTE, Jean-Marc, PARPETTE, Chantal (2011). Le Français sur objectif universitaire. France : PUG. QUEFFÉLEC, Ambroise, DERRADJI, Yacine (2002). Le Français en Algérie. Lexique et dynamique des langues. Bruxelles : De Boeck et Larcier s.a, éditions Duculot.

\section{NOTES}

1. L'enseignement peut être aussi de type informel, assuré par la famille ou certaines situations de communications informelles qui concernent aussi bien les scolarisés que des adultes.

2. Il est à préciser qu'à l'université algérienne, on assiste à un enseignement arabophone et un enseignement francophone. En effet, la majorité des cursus universitaires tels que les sciences humaines et sociales sont arabisés, autrement dit, la langue d'enseignement, d'accès au savoir de la spécialité est l'arabe. Cela dit, malgré l'arabité de l'ensemble de ces filières, l'enseignement d'une deuxième langue vivante étrangère est obligatoire, et il s'agit en général de la langue française. Quant à l'enseignement en français, ce dernier ne concerne qu'une partie des disciplines scientifiques : la biologie, les sciences médicales, les sciences véto-agro-alimentaires, et certaines filières techniques (génie civil). C'est à travers la langue française que va se faire dans le supérieur la transmission du savoir scientifique et technique.

3. Afin d'adopter un système de diplôme commun, de favoriser la mobilité des étudiants et leur assurer un accès au monde du travail dans toute l'Europe, l'Algérie a également décidé d'introduire le régime LMD au sein de ses différents établissements universitaires. 
4. Dès la $2^{\text {ème }}$ année ou $3^{\text {ème }}$ année d'étude, un enseignement d'anglais est également proposé. Il vise le plus souvent l'accès à l'information et à la documentation scientifique.

\section{RÉSUMÉS}

Dans le contexte algérien, l'enseignement du primaire jusqu'au secondaire est dispensé en arabe et ce n'est qu'à partir de la troisième année de l'enseignement primaire que l'apprentissage du français apparaît dans les programmes. À l'université, cette arabisation n'a été poursuivie que dans certaines disciplines universitaires, sciences sociales, économiques, commerciales... Et la plupart des filières scientifiques et techniques telles que médecine, sciences vétérinaires, architecture... enseignent en français. Le français devient donc langue d'accès à la formation scientifique. Cela signifie qu'à l'université algérienne, on dispose donc d'un enseignement arabophone et d'un enseignement francophone. Cet état de fait est souvent problématique pour les nouveaux arrivants à l'université qui se retrouvent contraints de poursuivre des études complexes dans une langue que très souvent ils ne maîtrisent pas suffisamment. C'est la raison pour laquelle notre article se propose de voir le type de palliatif à offrir aux nouveaux inscrits essentiellement ceux des disciplines scientifiques et techniques en français, en l'occurrence, les sciences vétérinaires, en début de leur formation universitaire. Il s'agit évidemment d'un enseignement de français (présenté sous forme de cours de langue) au-delà de l'enseignement en français (qui concerne les cours de la discipline). La mise en place du palliatif pour une formation linguistique s'inscrit dans le cadre du FOU (Français sur objectif universitaire).

In the Algerian context, education from primary to secondary is taught in Arabic and it is not until the third primary year learning French appears in programs. At university, this Arabization has been prosecuted in some academic disciplines, social sciences, economic, trade ... And most of the scientific and technical fields such as medicine, veterinary science, architecture ... teach in French. The French language is therefore access to scientific training. This means that the Algerian university, we therefore have a teaching Arabic and a francophone education. This fact is often problematic for newcomers to the university who are forced to pursue complex studies in a language they often have insufficient knowledge. That is why our paper aims to see the kind of palliative to offer new registrants essentially those of science and technology in French, namely veterinary sciences, early in their academic training. This is obviously a French education (in the form of language courses) beyond instruction in French (which concerns during the discipline). The implementation of the palliative for language training is part of the FOU (French on academic objective).

\section{INDEX}

Mots-clés : statut, FOS (Français sur objectif spécifique), FOU (Français sur objectif universitaire), approche par modélisation, compétence scripturale

Keywords : status, FOS (French on specific objective), FOU (French on academic objective), scriptural competence, approach modeling 


\section{AUTEUR}

\section{LAMIA BOUKHANNOUCHE}

Université de Blida 2

lamiafdz[at]yahoo.fr 